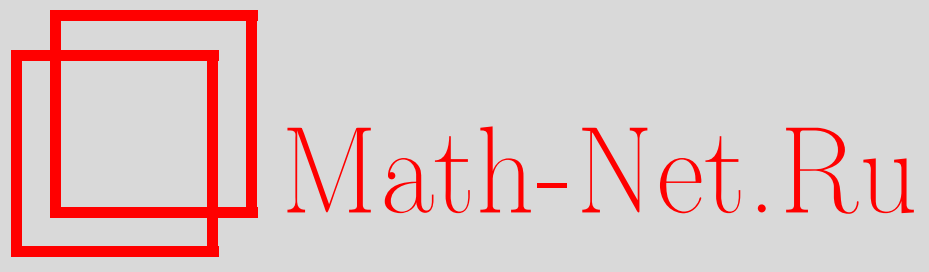

Д. А. Шабанов, О нижней оценке функции Ван дер Вардена, Матем. заметки, 2010, том 87, выпуск 6, 951-953

DOI: https://doi.org/10.4213/mzm8749

Использование Общероссийского математического портала Math-Net.Ru подразумевает, что вы прочитали и согласны с пользовательским соглашением http://www . mathnet.ru/rus/agreement

Параметры загрузки:

IP: 54.166 .219 .16

26 апреля 2023 г., 10:24:16

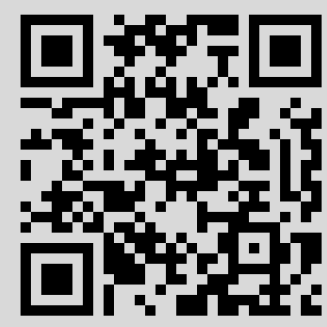




\section{О нижней оценке функции Ван дер Вардена}

\section{Д. А. Шабанов}

1. Введение и история задачи. В 1927 г. Ван дер Варден доказал (см. [1]) знаменитую теорему об арифметических прогрессиях. Данная теорема утверждает, что в любой раскраске множества цельх чисел $\mathbb{Z}$ в $r$ иветов найдется одноцветная арифметическая прогрессия сколь угодно большой длины. Теорема Ван дер Вардена является одним из фундаментальных результатов теории Рамсея. Ее эквивалентная формулировка звучит следующим образом.

Теорема 1 (Ван дер Варден, 1927). Для любых натуральных чисел $n \geqslant 3 u r \geqslant 2$ существует такое минимальное число $W(n, r)$, что для каждого натурального $N \geqslant$ $W(n, r)$ в любой $r$-цветной раскраске множества $\{1,2, \ldots, N\}$ найдется одноцветная арифметическая прогрессия длины $n$.

Функцию $W(n, r)$ из теоремы 1 принято называть функцией Ван дер Вардена. Оригинальное доказательство теоремы Ван дер Вардена дает очень слабые оценки $W(n, r)$ даже для $r=2$, из него лишь следует, что $W(n, 2) \leqslant A(n)$, где $A(n)$ - это функция Аккермана. Подробнее об этом можно прочитать в обзоре [2]. Наилучшая верхняя оценка в общем случае была получена Гауэрсом (см. [3]). Он доказал, что если $r \leqslant\left(\log _{2} \log _{2} N\right)^{c_{n}}$, где $c_{n}=2^{-2^{n+9}}$, то $W(n, r) \leqslant N$. В частном случае $n=3$ данная оценка была улучшена Грэмом и Шолимоши (см. [4]):

$$
W(3, r) \leqslant 2^{2^{c r}},
$$

где $c$ - некоторая абсолютная положительная константа.

Первая нетривиальная нижняя оценка для $W(n, r)$ была получена Эрдёшем и Радо в 1952 г. (см. [5]). Они доказали, что

$$
W(n, r) \geqslant \sqrt{2(n-1) r^{n-1}} .
$$

В дальнейшем эта оценка для больших (по сравнению с $n$ ) значений $r$ была улучшена Мозером в 1960 г. (см. [6]), который для некоторой абсолютной константы $C>0$ обосновал неравенство

$$
W(n, r) \geqslant n r^{C \ln r} .
$$

Результат Эрдёша и Радо был асимптотически улучшен Шмидтом (см. [7]). Он доказал существование такой абсолютной константы $c>0$, что для всех $n \geqslant 3, r \geqslant 2$ выполнено

$$
W(n, r) \geqslant r^{n-c \sqrt{n \ln n}} .
$$

В частном случае, когда $n$ - простое число, а $r=2$, Берлекамп установил (см. [8]) соотношение $W(n+1,2)>n 2^{n}$.

Последние наилучшие нижние оценки в задаче о $W(n, r)$ были получены с помощью применения вероятностных методов в теории гиперграфов. Напомним ряд определений. Гиперграфом $H$ называется пара $H=(V, E)$, где $V$ - некоторое конечное множество, называемое множеством вершин, а $E$ - совокупность различных подмножеств множества $V$, которые называются ребрами гиперграфа. Гиперграф является $n$-равномерным,

Работа выполнена при поддержке Российского фонда фундаментальных исследований (грант № 09-01-00294) программы "Ведущие научные школы" (грант № НШ-8784.2010.1) и гранта Президента РФ (грант № MK-3429.2010.1).

(C) Д. А. ШАванов, 2010 
если каждое его ребро состоит ровно из $n$ вершин. Раскраска вершин гиперграфа называется правильной, если в этой раскраске все ребра гиперграфа являются неодноцветными. Хроматическим числом (обозначение $\chi(H)$ ) гиперграфа $H$ называется минимальное число цветов, требуемое для правильной раскраски множества вершин. Если $\chi(H) \leqslant r$, то $H$ называется $r$-раскрашиваемым.

В знаменитой совместной работе Эрдёша и Ловаса (см. [9]) был установлен следующий результат, дающий достаточное условие $r$-раскрашиваемости $n$-равномерного гиперграфa.

Теорема 2 (Эрдёш, Ловас, 1973). Если степень каждой вершины n-равномерного гиперграфа $H$ не превосходит $r^{n-1} /(4 n)$, по $\chi(H) \leqslant r$.

Доказательство данной теоремы в существенной степени опиралось на известное утверждение теории вероятностей, называемое локальной леммой, которая как раз впервые и была получена в [9]. Следствием из теоремы 2 явилась оценка функции $W(n, r)$ :

$$
W(n, r) \geqslant \frac{r^{n-1}}{4 n}\left(1-\frac{1}{n}\right) .
$$

Очевидно, что оценка (3) асимптотически улучшает предыдущий результат Шмидта (2). В 1990 г. Сабо получил [10] наилучшую на сегодняшний день нижнюю оценку $W(n, r)$ в случае $r=2$. Он доказал, что для любого $\varepsilon>0$ существует такое натуральное число $n_{0}=n_{0}(\varepsilon)$, что для всех $n>n_{0}$ выполняется неравенство $W(n, 2) \geqslant 2^{n} n^{-\varepsilon}$. Таким образом, фактически Сабо обосновал неравенство вида

$$
W(n, 2) \geqslant 2^{n} n^{-|o(1)|}
$$

2. Новые результаты. Основным результатом данной работы является новая асимптотическая нижняя оценка функции Ван дер Вардена $W(n, r)$. Сформулируем сначала улучшение теоремы 2 Эрдёша и Ловаса.

Теорема 3. Если степень каждой вершины $n$-равномерного гиперграфа $H$ не превосходит величинъ

$$
d=\left(\frac{\sqrt{6}-2}{4}\right) \frac{r^{n-1}}{\sqrt{n \ln n}}
$$

$m o \chi(H) \leqslant r$.

ДокАЗАТЕЛЬСтво данной теоремы основано на применении локальной леммы, а также метода случайной раскраски вершин гиперграфа в $r$ цветов, который был предложен в работе [11].

Из теоремы 3 вытекает следующая нижняя оценка функции Ван дер Вардена $W(n, r)$.

Теорема 4. Для любых $n \geqslant 3, r \geqslant 2$ выполнено неравенство

$$
W(n, r) \geqslant\left(\frac{\sqrt{6}-2}{4}\right) \frac{r^{n-1}}{\sqrt{n \ln n}}\left(1-\frac{1}{n}\right) .
$$

Несложно понять, что полученная нами оценка (5) улучшает оценку Эрдёша и Ловаса (3) при $n \geqslant 15$. Но, в то же время, (5) асимптотически хуже результата Сабо (4), если $r=2$. В случае же, когда параметр $r$ экспоненциально велик по сравнению с $n$ $(n=o(\ln r))$, наилучшей остается оценка Мозера (1). В следующем пункте мы выведем теорему 4 из теоремы 3. 
3. Доказательство нижней оценки функции Ван дер Вардена. Итак, пусть заданы $n \geqslant 3$ и $r \geqslant 2$. Пусть также $N \geqslant n-$ произвольное натуральное число. Рассмотрим гиперграф

$$
H_{N}=\left(V_{N}, E_{N}\right), \quad V_{N}=\{1,2, \ldots, N\},
$$

а $E_{N}$ - это совокупность всех арифметических прогрессий длины $n$, которые содержатся в множестве $V_{N}$. Ясно, что $H_{N}-n$-равномерный гиперграф. Из построения также вытекает, что для каждого $N$ гиперграф $H_{N}$ вложен в гиперграф $H_{N+1}$ (в том смысле, что $V_{N} \subset V_{N+1}$ и $\left.E_{N} \subset E_{N+1}\right)$. Тогда

$$
\chi\left(H_{N}\right) \leqslant \chi\left(H_{N+1}\right) .
$$

Далее, из определения величины $W(n, r)$ следует, что

$$
W(n, r)=\min \left\{N: \chi\left(H_{N}\right)>r\right\} .
$$

Рассмотрим произвольное число $x \in V_{N}$. Сколько арифметических прогрессий длины $n$ из множества $\{1,2, \ldots, N\}$ содержат $x$ ? Каждая такая прогрессия однозначно определяется порядковым номером $x$ в этой прогрессии, а также разностью прогрессии. Порядковый номер может меняться от 1 до $n$, а разность - от 1 до $(N-1) /(n-1)$. Таким образом, для любой вершины $x \in V_{N}$ ее степень в гиперграфе $H_{N}$ не превосходит $n(N-1) /(n-1)$. Поэтому из теоремы 3 следует, что если выполняется неравенство

$$
N \leqslant\left(\frac{\sqrt{6}-2}{4}\right) \frac{r^{n-1}}{\sqrt{n \ln n}}\left(1-\frac{1}{n}\right)+1,
$$

то $\chi\left(H_{N}\right) \leqslant r$. Из данного соотношения, а также из (6) и (7) получаем окончательную оценку (5) функции Ван дер Вардена. Теорема 4 доказана.

\section{СПИСОК ЦИТИРОВАННОЙ ЛИТЕРАТУРЫ}

[1] В.L. van der Waerden, Nieuw Archief, 15 (1927), 212-216. [2] И.Д. Шкредов, УMH, 61:6 (2006), 111-178. [3] W. T. Gowers, Geom. Funct. Anal., 11:3 (2001), 465-588. [4] R. Graham, J. Solymosi, Topics in Discrete Mathematics, Algorithms Combin., 26, Springer-Verlag, Berlin, 2006, 129-132. [5] P. Erdős, R. Rado, Proc. London Math. Soc. (3), 2 (1952), 417-439. [6] L. Moser, Canad. Math. Bull., 3 (1960), 23-25. [7] W. M. Schmidt, Duke Math. J., 29:1 (1962), 129-140. [8] E. R. Berlekamp, Canad. Math. Bull., 11 (1968), 409-414. [9] P. Erdős, L. Lovász, Infinite and Finite Sets, v. II (Keszthely, 1973), Colloq. Math. Soc. Janos Bolyai, 10, North Holland, Amsterdam, 1975, 609-627. [10] Z. Szabó, Random Structures Algorithms, 1:3 (1990), 343-360. [11] Д. А. Шабанов, Матем. заметки, 85:6 (2009), 951-954.

\section{Д. А. Шабанов}

